\title{
Convex combinations of adaptive algorithms for blind equalization of QAM signals
}

\author{
Magno T. M. Silva and João Mendes Filho
}

\begin{abstract}
Based on concurrent algorithms and on the convex combination of one slow and one fast CMA (Constant Modulus Algorithm), we propose a convex combination of two blind equalizers adapted respectively by CMA and the modified SDD (Soft Decision-Directed) algorithm for recovering of QAM (Quadrature Amplitude Modulation) signals. For high signal-to-noise ratio, the performance of the proposed scheme is, in the worst case, as good as that of the best of its components, being better than both of them in some situations. Since the considered algorithms are derived from different criteria, the mixing parameter is updated for minimizing the decision-directed cost function.
\end{abstract}

Keywords-Adaptive filtering, blind equalization, QAM signals, convex combination, Constant Modulus Algorithm.

\section{INTRODUCTION}

Blind equalizers are used in modern digital communication systems to remove intersymbol interference introduced by dispersive channels. The Constant Modulus Algorithm (CMA) [1] is the most popular for the adaptation of finite impulse response (FIR) equalizers. It is widely employed even for nonconstant modulus constellations as the $M$-QAM (Quadrature Amplitude Modulation) signalling with $M>4$ [2]-[4]. Although it presents an advantage of having simple computational complexity, its main drawback is the possible convergence to undesirable local minima. In this case, it may achieve just a moderate level of Mean-Square Error (MSE) after convergence [2]-[4]. To reduce the residual CMA steadystate MSE, an alternative is to switch to the Decision-Directed (DD) mode. However, to ensure a successful transfer from CMA to DD algorithm, the CMA steady-state MSE should be sufficiently low, which may not always be reached by CMA [2]-[4].

Another blind algorithm for the adaptation of FIR equalizers is the Soft Decision-Directed (SDD) algorithm [4]. It was originally derived in [5] for 4-QAM constellation and extended to $M$-QAM $(M \geq 4)$ in [6]. Using a multi-stage procedure [6], its computational complexity is always equivalent to the 4-QAM case, which makes its implementation feasible even for $M \gg 4$. As pointed out in [3], [4], its main drawback is that the adaptation process requires $L$-stage switchings, where $L=\log _{2}(M) / 2$, and each adaptation stage needs a different set of parameters.

In order to improve CMA performance, some attention has been given to the concurrent adaptation. A concurrent

Magno T. M. Silva is with Electronic Systems Engineering Department, Escola Politécnica, Universidade de São Paulo, São Paulo, SP, Brazil. João Mendes Filho is with Programa de Pós-Graduação em Engenharia Elétrica, Universidade Presbiteriana Mackenzie, São Paulo, SP, Brazil. E-mails: magno@lps.usp.br, joao.mendes@uol.com.br. algorithm formed by CMA and the DD algorithm for $M$ QAM signaling was introduced in [2]. Later, considering the last stage of the SDD algorithm, a modification in the concurrent scheme of [2] was proposed in [3], [4]. The resulting concurrent algorithm, denoted by SDD-CMA, has lower computational complexity and faster convergence rate than DD-CMA [4]. It does not require difficult tuning of parameters, since only the last stage of SDD is considered (the first $L-1$ stages are replaced by simple comparisons) [3].

Although these algorithm combinations represent an improvement in the equalization performance, it depends on the good behavior of each component algorithm. If, for example, CMA presents a high level of steady-state MSE due to an inadequate choice of step-size, the concurrent algorithm will perform badly. Moreover, only the last stage of SDD does not present an adequate individual performance for $M$-QAM signalling with $M>16$. In this case, the blind multi-stage procedure of [6] should be used to ensure a correct clustering separation. To improve the overall performance in such situations, each component filter could be individually adapted and their outputs mixed together considering an adaptive convex combination.

Adaptive convex combinations of algorithms have been proposed in the literature. A convex combination of one fast and one slow LMS (Least Mean-Square) filter was introduced in [7] and analyzed in [8]. Furthermore, it was shown that this structure presents a worst case performance as good as that of the best of its components, and outperforms both of them in some situations [8]. In this scheme, each component filter is adapted separately and the mixing parameter is adjusted online by means of a nonlinear rule [8]. It was also extended for blind equalization purposes in [9] and [10], considering respectively the combination of one fast and one slow CMA (CCMA) and the combination of CMA with the ShalviWeinstein Algorithm.

In order to avoid the difficult adjustment of the parameters of the multi-stage SDD algorithm, we consider only its last stage and replace the first $L-1$ stages by simple comparisons as [3]-[4]. However, to improve its performance for $M>16$, we propose a modification in such algorithm, resulting in the Modified SDD (MSDD) algorithm. Then, inspired in [7][9], we propose a convex combination of one CMA and one MSDD (C-MSDD-CMA). The proposed combination should be able to perform as well as the best of its components, outperforming both of them and the concurrent algorithm of [3] in some situations.

The paper is organized as follows. In Section II, the problem is formulated and CMA, SDD, SDD-CMA, and CCMA are 
revisited. The modification to use only the last stage of SDD algorithm independently of $M$ is also presented. In Section III, the convex combination of one MSDD and one CMA is introduced. Simulation results are presented in Section IV, where the proposed scheme is compared to the concurrent SDD-CMA and the convex combination of two CMAs. To close the paper, conclusions are shown in Section V.

\section{BLIND ADAPTATION OF FIR EQUALIZERS}

A simplified block diagram of a baseband communication system is depicted in Figure 1 [11]. The signal $a(n)$ is transmitted through an unknown channel, whose model is constituted by an FIR filter $H(z)$ and Additive White Gaussian Noise (AWGN) $\eta(n)$. The received signal $u(n)$ is a distorted version of $a(n)$, corrupted by intersymbol interference and noise. It passes through an FIR filter, whose output is given by the inner product $y(n)=\mathbf{u}^{T}(n) \mathbf{w}$, where $\mathbf{u}(n)=\left[\begin{array}{llll}u(n) & u(n-1) & \cdots & u(n-M+1)\end{array}\right]^{T}$ and $\mathbf{w}=$ $\left[\begin{array}{llll}w_{0} & w_{1} & \cdots & w_{M-1}\end{array}\right]^{T}$ are the input regressor vector and the coefficient vector respectively, $M$ is the filter length, and the superscript $T$ stands for the transpose of a vector. After a phase correction, the signal enters to the decision device and a delayed estimate of the transmitted sequence is obtained, i.e., $\hat{a}(n-\Delta)$, being $\Delta$ a positive integer.

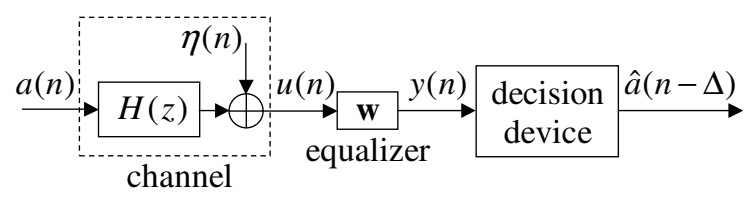

Fig. 1. A simplified communication system with an FIR equalizer.

\section{A. Constant Modulus and Phase Tracking algorithms}

The constant modulus cost function is given by [1]

$$
J_{C M}=\mathrm{E}\left\{\left(|y(n)|^{2}-R_{2}^{a}\right)^{2}\right\},
$$

where $R_{2}^{a}=\mathrm{E}\left\{|a(n)|^{4}\right\} / \mathrm{E}\left\{|a(n)|^{2}\right\}$ and $\mathrm{E}\{\cdot\}$ stands for the expectation operator. Taking the instantaneous gradient of (1) with respect to $\mathrm{w}$, we obtain the CMA update rule, that is,

$$
\mathbf{w}(n)=\mathbf{w}(n-1)-\mu_{c} e(n) \mathbf{u}^{*}(n)
$$

where

$$
e(n)=y(n)\left(|y(n)|^{2}-R_{2}^{a}\right),
$$

$y(n)=\mathbf{u}^{T}(n) \mathbf{w}(n-1), \mu_{c}$ is the step-size, and the superscript $*$ stands for the conjugate.

Since CMA is insensible to a random phase rotation, it is common to include a phase correction algorithm. After a successful convergence, the output of the equalizer can be modeled by

$$
y(n) \approx \mathrm{e}^{j \varphi} a(n-\Delta)+\nu(n),
$$

where $\varphi \in[0,2 \pi[$ represents the phase rotation and $\nu(n)$ is a complex AWGN sample. The phase rotation must be compensated before the decision device. The literature contains several blind phase recovery algorithms (see e.g. [12], [13], and the references therein), but for simplicity we assume in this paper the Phase Tracking Algorithm (PTA) [11], [14], which provides the following phase update equation

$$
\varphi(n+1)=\varphi(n)+\mu_{\varphi} \operatorname{Im}\left\{\bar{y}(n) \hat{a}^{*}(n-\Delta)\right\},
$$

where $\bar{y}(n)=y(n) e^{-j \varphi(n)}, \mu_{\varphi}$ is the step-size, and $\operatorname{Im}\{\cdot\}$ stands for the imaginary part of a complex number.

\section{B. The Soft Decision Directed Algorithm}

The SDD algorithm is a stochastic gradient-type algorithm derived from the minimization of the cost function

$$
J_{M A P}=-\mathrm{E}\left\{\rho \log \left[\hat{p}_{i}(y(n))\right]\right\},
$$

where $\rho$ is a parameter which depends on the noise power, and $\hat{p}_{i}(y(n))$ is a local approximation to the a posteriori probability density function (p.d.f.) of $y(n)$. Since the p.d.f. of $y(n)$ depends on the difference among it and the QAM constellation symbols, the random phase rotations (non-multiple of $\pi / 2$ ) are automatically corrected ${ }^{1}$. Thus, a phase recovery algorithm (like PTA) is not necessary, $y(n)=\bar{y}(n)$, and $y(n)$ can be modeled by (4) with $\varphi=0$.

Using such model, $y(n)$ can be approximated by $M$ Gaussian clusters, where the complex AWGN $\nu(n)=\nu_{R}(n)+$ $j \nu_{I}(n)$ is such that $\mathrm{E}\left\{\nu_{R}(n) \nu_{I}(n)\right\}=0$ and $\mathrm{E}\left\{\nu_{R}^{2}(n)\right\}=$ $\mathrm{E}\left\{\nu_{I}^{2}(n)\right\}=\rho$, being $\nu_{R}$ and $\nu_{I}$ the real and imaginary parts of $\nu$, respectively. Hence, the a posteriori p.d.f. of $y(n)$ can be approximated by

$$
p(y(n)) \approx \sum_{k=1}^{K} \sum_{l=1}^{K} \frac{P_{k l}}{2 \pi \rho} \exp \left[-\frac{\left|y(n)-a_{k l}\right|^{2}}{2 \rho}\right],
$$

where $a_{k l}$ takes the value from the $M$-QAM symbol set defined by

$$
A=\left\{a_{k l}=(2 k-K-1)+j(2 l-K-1), \quad 1 \leq k, l \leq K\right\}
$$

with $K=\sqrt{M}$ and $P_{k l}$ the a priori probability of $a_{k l}$. For equiprobable symbol transmission $P_{k l}=1 / M, \quad 1 \leq k, l \leq$ $K$. The SDD algorithm was derived considering (7) and the stochastic gradient method in conjunction with a multi-stage procedure in the minimization of (6). Ideally, $\rho$ is the power of the real or imaginary part of $\nu(n)$. However, since the noise power is not known in practice, $\rho$ is a parameter which must be chosen. The adjustment of $\rho$ depends on each stage and may be a difficult task [6], [3].

To minimize the computational complexity, the complex plan was divided into $M / 4$ regular regions as indicated in Figure 2, with each region $A_{i}$ containing four symbols, that is, $A_{i}=\left\{a_{i m}, m=1,2,3,4\right\}$, being $i=1,2, \ldots, M / 4$. Defining $\varepsilon_{i m}(n) \triangleq y(n)-a_{i m}$, if the equalizer output is within the region $A_{i}$, a local approximation to the a posteriori p.d.f. of $y(n)$ is

$$
\hat{p}_{i}(y(n)) \approx \sum_{m=1}^{4} \frac{1}{8 \pi \rho} \exp \left[-\frac{\left|\varepsilon_{i m}(n)\right|^{2}}{2 \rho}\right],
$$

\footnotetext{
${ }^{1}$ Phase rotation multiple of $\pi / 2$ can be avoided by using differential modulation [11].
} 
being that the a priori probability has been set to $1 / 4$. Then, using (8) and replacing the $L-1$ stages by simple comparisons, a simplified version of the SDD algorithm was derived in [3] and its update equation is given by

$$
\mathbf{w}(n)=\mathbf{w}(n-1)-\mu_{d} \xi(n) \mathbf{u}^{*}(n),
$$

where

$$
\xi(n)=\frac{\sum_{m=1}^{4} \exp \left[-\frac{\left|\varepsilon_{i m}(n)\right|^{2}}{2 \rho}\right] \varepsilon_{i m}(n)}{\sum_{m=1}^{4} \exp \left[-\frac{\left|\varepsilon_{i m}(n)\right|^{2}}{2 \rho}\right]}
$$

and $\mu_{d}$ is the step-size. In this case, to ensure a proper separation of the clusters, $\rho$ should be lower than the half of the minimum distance between two neighboring constellation symbols [3].

As indicated in (10), the SDD adaptation needs to compute only $4 \exp (\cdot)$ at each iteration, which can be implemented through a table. The task of $M$-QAM equalization, where $M=2^{2 L}$, is achieved with $L$ stages, being that $L-1$ stages consist in only $2(L-1)$ comparisons to identify the region $A_{i}$, which does not cause a significant increase in computational complexity. Thus, the algorithm's complexity is always equivalent to the minimum complexity of the 4-QAM case [4]. Considering for example 64-QAM, the complex plan is divided in $64 / 4=16$ regions. Each region must contain 4 symbols as shown in Figure 2. Suppose that the equalizer output is $6.0+j 6.5$. The first stage consists in classifying the output into the 4 quadrants of the complex plan. In the example, the output is in the first quadrant because its real and imaginary parts are positive. At the second stage, the output must be classified into other 4 regions and at the last stage $\xi(n)$ can be calculated using the four symbols $a_{i m}, m=1,2,3,4$ of the final region $A_{i}$.

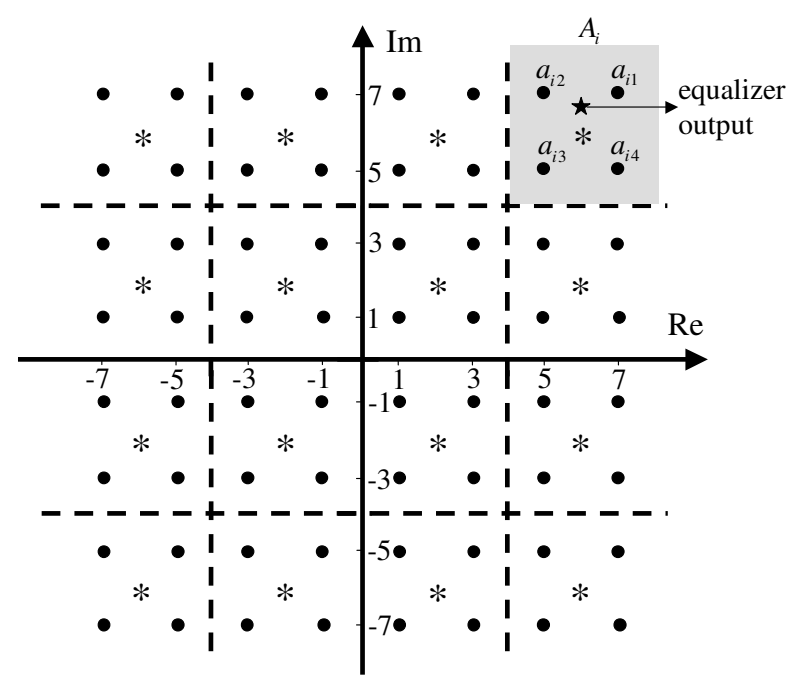

Fig. 2. Illustration of local regions for SDD adaptation and 64-QAM; the center of each local region is indicated by $*$, and the constellation symbols are represented by $\bullet$

\section{The Modified SDD}

Maintaining only the last stage, to ensure a correct behavior of the SDD algorithm even for $M>16$, the error $\xi(n)$ of (10) is multiplied by the absolute value of the center of the local region $A_{i}$ at each iteration. Thus, if the region $A_{i}$ is the one indicated in Figure 2, for example, $\xi(n)$ must be multiplied by $|6+j 6|=6 \sqrt{2}$. This modified algorithm is named MSDD. The modification can be interpreted as a simple adjust in the SDD step-size. Since the local region $A_{i}$ is different at each iteration, (9) needs to be compensated to ensure a correct equalization for all regions of the complex plan. At an additional cost of one single multiplication per iteration, we have observed (by means of exhaustive simulations) that the MSDD algorithm outperforms SDD in the equalization of QAM signals. We should notice that for 16-QAM, since the centers of the four regions $A_{i}$ are equal to $c=2 \sqrt{2}$, MSDD with step-size $\mu_{d}$ reduces to SDD with step-size $c \mu_{d}$. For 4QAM, since the center of the single region coincides with the origin of the complex plan, to ensure a correct behavior of MSDD, we assume $c=1$. Then, in this case, MSDD and SDD are the same algorithms.

Although the MSDD algorithm presents a steady-state performance better than SDD and sometimes much better than CMA, it suffers from a slow convergence. Thus, its combination with CMA allows a better performance mainly during the convergence, as shown by the simulations of Section IV.

\section{The concurrent SDD-CMA}

The concurrent algorithm of [3], denoted by SDD-CMA, has the following update equation

$$
\mathbf{w}(n)=\mathbf{w}_{c}(n)+\mathbf{w}_{d}(n),
$$

where $\mathbf{w}_{c}$ and $\mathbf{w}_{d}$ are the coefficient vectors updated by CMA and SDD algorithm, that is, by eqs. (2) and (9), respectively. As the SDD algorithm, SDD-CMA does not need a phase recovery algorithm for rotations non-multiple of $\pi / 2$. It is relevant to note that the errors (3) and (10), used to update respectively $\mathbf{w}_{c}(n)$ and $\mathbf{w}_{d}(n)$, depend on the coefficients of previous time instants of both algorithms, since they depend on $y(n)$ and $y(n)=\mathbf{u}^{T}(n) \mathbf{w}(n-1)=$ $\mathbf{u}^{T}(n)\left[\mathbf{w}_{c}(n-1)+\mathbf{w}_{d}(n-1)\right]$. Consequently, if one of both algorithms does not perform well, the concurrent will also present a bad performance.

\section{E. Convex combination of two CMAs}

A convex combination of one fast and one slow CMA was proposed in [9] and is depicted in Figure 3. In this scheme, the overall coefficient vector is given by

$$
\mathbf{w}(n-1)=\lambda(n) \mathbf{w}_{1}(n-1)+[1-\lambda(n)] \mathbf{w}_{2}(n-1),
$$

where $\mathbf{w}_{1}$ and $\mathbf{w}_{2}$ are updated by (2), considering the stepsizes $\mu_{c 1}$ and $\mu_{c 2}$ respectively, with $\mu_{c 1}>\mu_{c 2}$. Similarly, the output of the overall equalizer is given by

$$
y(n)=\lambda(n) y_{1}(n)+[1-\lambda(n)] y_{2}(n),
$$

where $y_{i}(n), i=1,2$ are the outputs of the equalizers, i.e., $y_{i}(n)=\mathbf{u}^{T}(n) \mathbf{w}_{i}(n-1), \mathbf{u}(n)$ is the common regressor 
vector, and $\mathbf{w}_{i}(n-1)$ are the coefficient vectors of each component equalizer. The mixing parameter $\lambda(n)$ is modified via a sigmoidal function and the auxiliary variable $\alpha(n-1)$ [7]-[9], that is,

$$
\lambda(n)=\operatorname{sgm}[\alpha(n-1)]=\frac{1}{1+e^{-\alpha(n-1)}},
$$

with $\alpha(n)$ being updated as

$$
\alpha(n)=\alpha(n-1)-\mu_{\alpha} e_{\alpha}(n) \lambda(n)[1-\lambda(n)],
$$

where

$$
e_{\alpha}(n)=\left(|y(n)|^{2}-R_{2}^{a}\right) \operatorname{Re}\left\{y(n)\left[y_{1}(n)-y_{2}(n)\right]^{*}\right\},
$$

$\mu_{\alpha}$ is the step-size, and $\operatorname{Re}\{\cdot\}$ denotes the real part of a complex number. Equation (15) was obtained in [9], using a stochastic gradient rule to minimize (1). The auxiliary variable $\alpha(n)$ is used to keep $\lambda(n)$ in the interval $[0,1]$. A drawback of this scheme is that $\alpha(n)$ stops updating whenever $\lambda(n)$ is close to 0 or 1 . To avoid this, [9], [8] suggest that $\alpha(n)$ be restricted (by simple saturation) to lie inside a symmetric interval $\left[-\alpha^{+}, \alpha^{+}\right]$.

Since CMA does not recover the signal phase, PTA (Eq. (5)) can be used in conjunction with each component equalizer.

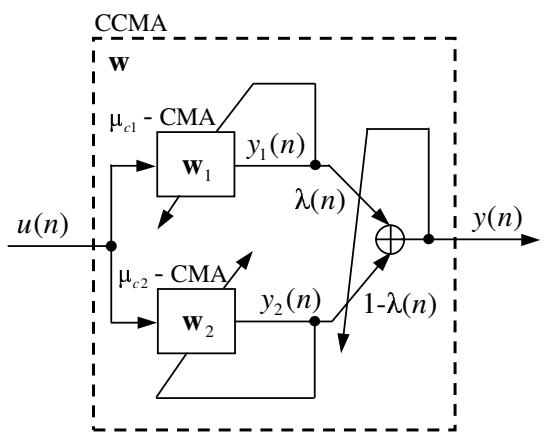

Fig. 3. Adaptive convex combination of two CMAs.

\section{Convex COMbination of OnE CMA AND ONE MSDD ALGORITHM}

The convex combination of one CMA and one MSDD is depicted in Figure 4. Since these algorithms minimizes different cost functions, the mixing parameter $\lambda(n)$ is adapted to minimize the decision-directed cost function, i.e.,

$$
J_{D}=\mathrm{E}\left\{\left|e_{d}(n)\right|^{2}\right\},
$$

where

$$
e_{d}(n)=y(n)-\hat{a}(n-\Delta)
$$

is the decision error. If $\lambda(n)$ was adapted to minimize (1) or (6), the combination would perform close to the algorithm related to the considered cost function. Thus, we choose (17), which is different from the cost functions of the combined algorithms.

As in the convex combination of two CMAs, we assume that $\lambda(n)$ is adapted via (14), i.e., using a sigmoidal function and the auxiliary variable $\alpha(n-1)$. Thus, we need to find a recurrent equation to update $\alpha(n-1)$. Using the gradient method to minimize the instantaneous version of $J_{D}$, that is, $\hat{J}_{D}(n)=\left|e_{d}(n)\right|^{2}$, we have

$$
\alpha(n)=\alpha(n-1)-\frac{\mu_{\alpha}}{2} \frac{\partial \hat{J}_{D}(n)}{\partial \alpha(n-1)} .
$$

Using (14) and the chain rule, we obtain

$$
\begin{aligned}
\frac{\partial \hat{J}_{D}(n)}{\partial \alpha(n-1)} & =\frac{\partial \hat{J}_{D}(n)}{\partial \lambda(n)} \frac{\partial \lambda(n)}{\partial \alpha(n-1)} \\
& =\frac{\partial \hat{J}_{D}(n)}{\partial \lambda(n)} \lambda(n)[1-\lambda(n)] .
\end{aligned}
$$

Rewriting $e_{d}(n)$ as a function of $\lambda(n)$, i.e.,

$$
e_{d}(n)=\lambda(n) y_{1}(n)+[1-\lambda(n)] y_{2}(n)-\hat{a}(n-\Delta) \text {, }
$$

we arrive at

$$
\begin{aligned}
\frac{\partial \hat{J}_{D}(n)}{\partial \lambda(n)} & =\frac{\partial\left[e_{d}^{*}(n) e_{d}(n)\right]}{\partial \lambda(n)} \\
& =\frac{\partial e_{d}^{*}(n)}{\partial \lambda(n)} e_{d}(n)+\frac{\partial e_{d}(n)}{\partial \lambda(n)} e_{d}^{*}(n) \\
& =2 \operatorname{Re}\left\{e_{d}(n)\left[y_{1}(n)-y_{2}(n)\right]^{*}\right\} .
\end{aligned}
$$

Now, replacing (22) in (20) and the result in (19), we obtain

$$
\alpha(n)=\alpha(n-1)-\mu_{\alpha} e_{\alpha, d}(n) \lambda(n)[1-\lambda(n)]
$$

where $e_{\alpha, d}(n)=\operatorname{Re}\left\{e_{d}(n)\left[y_{1}(n)-y_{2}(n)\right]^{*}\right\}$.

As in the convex combination of two CMAs, $\alpha(n)$ is restricted (by simple saturation) to lie inside the interval $\left[-\alpha^{+}, \alpha^{+}\right]$. Thus, the updating of $\alpha(n)$ never stops, even when $\lambda(n)$ is close to 0 or 1 . Furthermore, to avoid phase rotation, PTA is considered in conjunction with CMA, allowing an adequate convex combination.

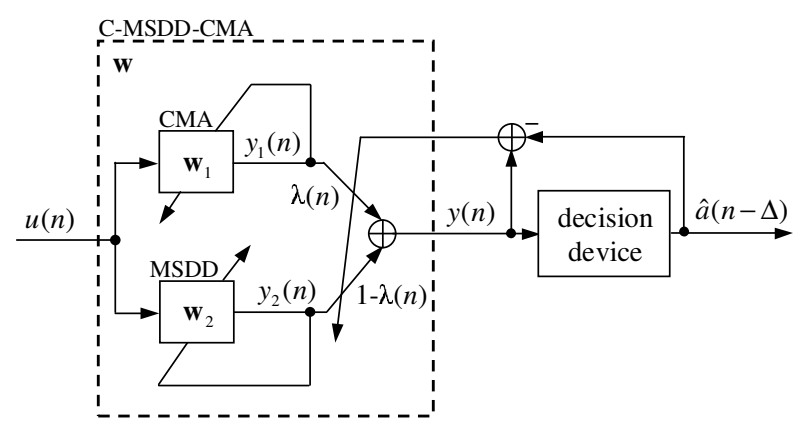

Fig. 4. Adaptive convex combination of one CMA and one MSDD.

\section{Simulation RESUlts}

In all the simulations, we assume: 64-QAM, the channels whose coefficients are in tables I and II, and equalizers with $M=23$ coefficients initialized with zero, except the $12^{\text {th }}$ one. 
TABLE I

A 22-TAP CHANNEL IMPULSE RESPONSE FROM [3].

\begin{tabular}{|c|c|c||c|c|c|}
\hline Tap & Real & Imag. & Tap & Real & Imag. \\
\hline 0 & 0.0145 & -0.0006 & 11 & 0.0294 & -0.0049 \\
\hline 1 & 0.0750 & 0.0176 & 12 & -0.0181 & 0.0032 \\
\hline 2 & 0.3951 & 0.0033 & 13 & 0.0091 & 0.0003 \\
\hline 3 & 0.7491 & -0.1718 & 14 & -0.0038 & -0.0023 \\
\hline 4 & 0.1951 & 0.0972 & 15 & 0.0019 & 0.0027 \\
\hline 5 & -0.2856 & 0.1896 & 16 & -0.0018 & -0.0014 \\
\hline 6 & 0.0575 & -0.2096 & 17 & 0.0006 & 0.0003 \\
\hline 7 & 0.0655 & 0.1139 & 18 & 0.0005 & 0.0000 \\
\hline 8 & -0.0825 & -0.0424 & 19 & -0.0008 & -0.0001 \\
\hline 9 & 0.0623 & 0.0085 & 20 & 0.0000 & -0.0002 \\
\hline 10 & -0.0438 & 0.0034 & 21 & 0.0001 & 0.0006 \\
\hline
\end{tabular}

TABLE II

A 3-TAP CHANNEL IMPULSE RESPONSE.

\begin{tabular}{|c|c|c|}
\hline Tap & Real & Imag. \\
\hline 0 & 0.3740 & -0.0556 \\
\hline 1 & 0.4689 & 0.7031 \\
\hline 2 & 0.3740 & -0.0556 \\
\hline
\end{tabular}

Figure 5-a) shows $J_{D}=\mathrm{E}\left\{\left|e_{d}(n)\right|^{2}\right\}$ estimated by the ensemble-average of 100 independent runs for the MSDD algorithm, CMA, and their convex combination. We consider the channel of Table I with a signal-to-noise ratio (SNR) of $40 \mathrm{~dB}$. To facilitate the visualization, the curves were filtered by a moving-average filter with 64 coefficients. The MSDD algorithm presents a initial convergence faster than that of CMA and stays at $J_{D} \approx-2 \mathrm{~dB}$ until $n=10^{5}$. Although CMA is initially slower than MSDD, it presents a better performance from $n=2 \times 10^{4}$ to $n=1.15 \times 10^{5}$. After that, MSDD reaches $J_{D} \approx-12 \mathrm{~dB}$, being better than CMA. Thus, the combination performs close to MSDD during the first $2 \times 10^{4}$ iterations, close to CMA from $n=2 \times 10^{4}$ to $n=10^{5}$, and switches back to MSDD from $n=1.25 \times 10^{5}$. It is relevant to note that from $n=10^{5}$ to $n=1.25 \times 10^{5}$, the combination outperforms both of its components. In Figure 5-b), we show $\mathrm{E}\{\lambda(n)\}$, confirming the previous observations.

In Figure 6, we compare the proposed convex combination with the concurrent SDD-CMA and with the combination of two CMAs (CCMA). C-MSDD-CMA presents a better performance than the other combinations during the first $2 \times 10^{4}$ iterations. However, it is slightly worse than the other combinations from $n=2 \times 10^{4}$ until $n=5 \times 10^{4}$ and presents an intermediate behavior from $n=5 \times 10^{4}$ until $n=10^{5}$. After that, it outperforms the other combinations, achieving $J_{D} \approx-12 \mathrm{~dB}$, while CCMA reaches $J_{D} \approx-8 \mathrm{~dB}$ and SDDCMA achieves $J_{D} \approx-7.2 \mathrm{~dB}$. The equalizer output signal constellations after convergence are shown in Figure 7. We can observe that the proposed convex combination presents the best performance, in regards of "eye-opening".

Figure 8-a) shows $J_{D}$ estimated by the ensemble-average of 70 independent runs for the MSDD algorithm, CMA, and their convex combination. At $n=2 \times 10^{5}$, the channel is changed
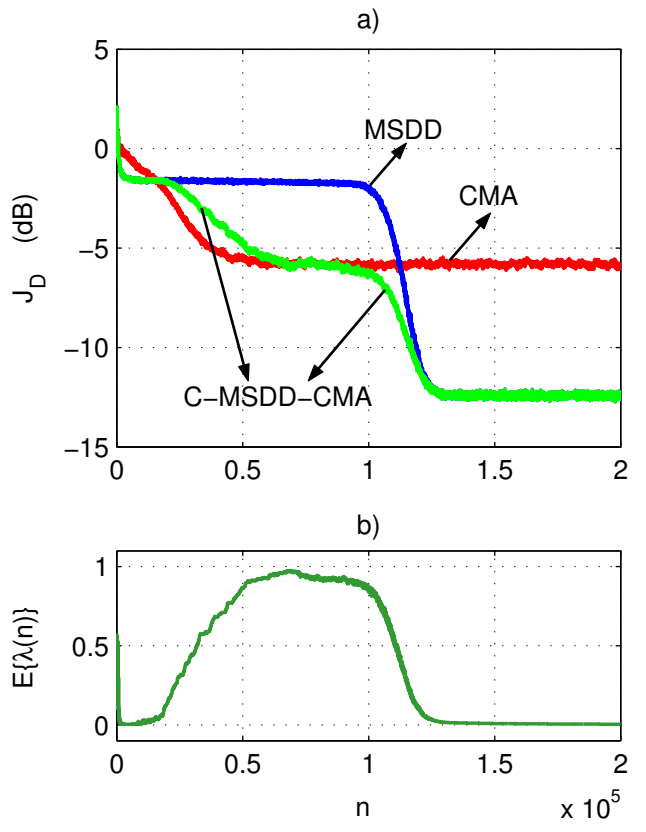

Fig. 5. a) Ensemble-average of the squared absolute value of the decision error for a) MSDD $\left(\mu_{d}=10^{-5}, \rho=0.6\right)$, CMA $\left(\mu_{c}=5 \times 10^{-7}\right.$, $\left.\mu_{\varphi}=10^{-3}\right)$ and their convex combination C-MSDD-CMA $\left(\mu_{\alpha}=2\right.$, $\left.\alpha^{+}=4\right) ; M=23$; 64-QAM, channel of Table I; SNR $=40 \mathrm{~dB}$; mean of 100 independent runs. b) Ensemble-average of $\lambda(n)$.

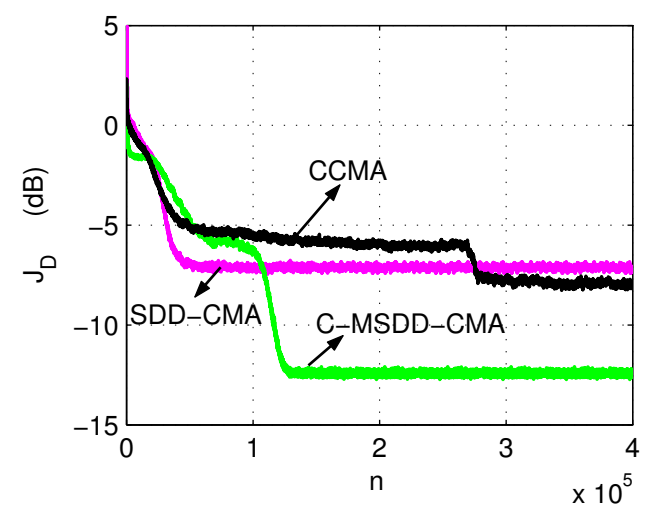

Fig. 6. a) Ensemble-average of the squared absolute value of the decision error for C-MSDD-CMA $\left(\mu_{c}=5 \times 10^{-7}, \mu_{\varphi}=10^{-3}, \mu_{d}=10^{-5}\right.$, $\left.\rho=0.6, \mu_{\alpha}=2, \alpha^{+}=4\right)$, SDD-CMA $\left(\mu_{c}=5 \times 10^{-7}, \mu_{d}=10^{-5}\right.$, $\rho=0.6)$, CCMA $\left(\mu_{1}=5 \times 10^{-7}, \mu_{2}=10^{-7}, \mu_{\alpha}=0.8, \mu_{\varphi}=10^{-3}\right.$, $\left.\alpha^{+}=4\right) ; M=23$; 64-QAM; channel of Table I; SNR $=40 \mathrm{~dB}$; mean of 100 independent runs.

from that of Table II to that of Table I. We assume SNR = $35 \mathrm{~dB}$ and $\mu_{\alpha}=5$. We can observe that the C-MSDD-CMA is close to MSDD in the steady-state. However, during the convergence, it presents an intermediate performance between those of CMA and MSDD. Through exhaustive simulations, we observed that the switching between its component algorithms depends on the signal-to-noise ratio. For low SNR $(<35 \mathrm{~dB})$, the decision error is high and the mixing parameter does not work properly in the convergence, which leads the combination to present a mean-square performance worse than one of its components. Figure 8-b) shows $\mathrm{E}\{\lambda(n)\}$, which is always smaller than 1 . This means that the combination does not perform like CMA in the mean-square sense. 
a) C-MSDD-CMA

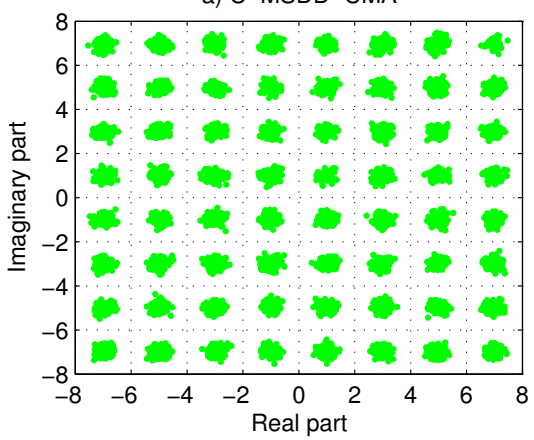

b) SDD-CMA

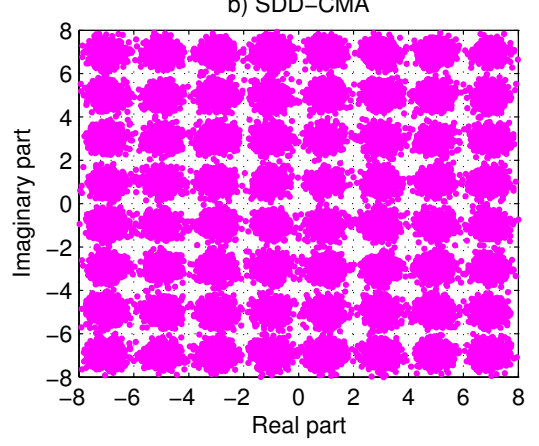

c) CCMA

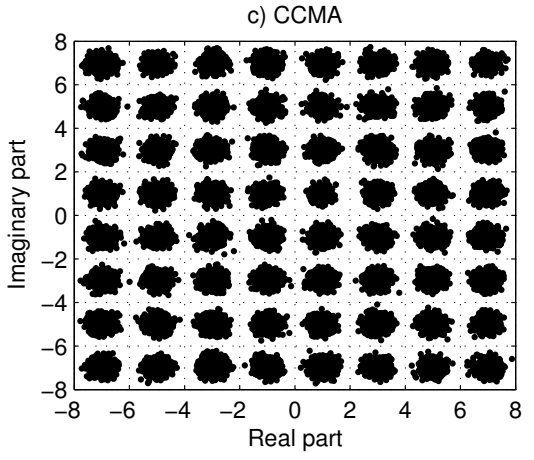

Fig. 7. Equalizer output signal constellations after convergence for a) CMSDD-CMA, b) SDD-CMA, and c) CCMA (the parameters of the algorithms are the same shown in the legend of Figure 6).

\section{CONCLUSIONS}

We proposed a modification to the SDD algorithm in order to use only its last stage in the recovering of $M$-QAM signals. Then, we combined the modified SDD with CMA to take advantage of their different convergence and steadystate behaviors. Through simulations, we verified that when the signal-to-noise ratio is higher than $35 \mathrm{~dB}$ the scheme seems to be universal, performing as the best component filter and being better than both of them in some situations. Furthermore, it can present a better performance than those of other algorithm combinations contained in the literature. However, for low SNR the minimization of decision directed cost function does not seem to be the most adequate to update the mixing parameter. Further work should imply different forms to update this parameter.

\section{REFERENCES}

[1] D. N. Godard, "Self-recovering equalization and carrier tracking in two dimensional data communication system," IEEE Transactions on a)

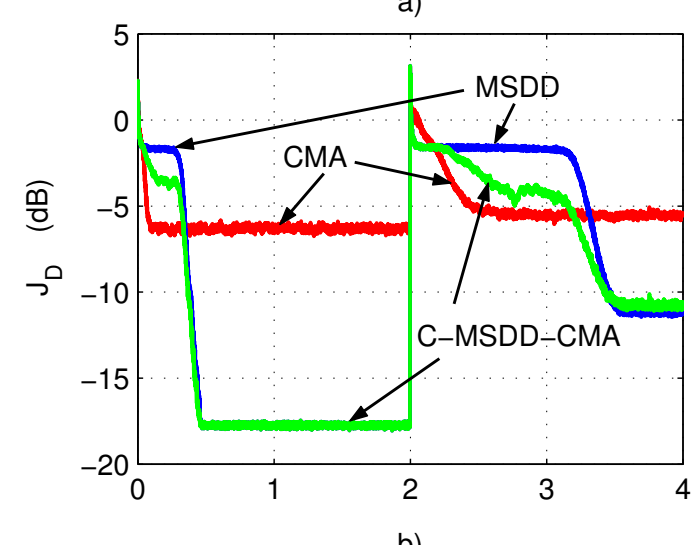

b)

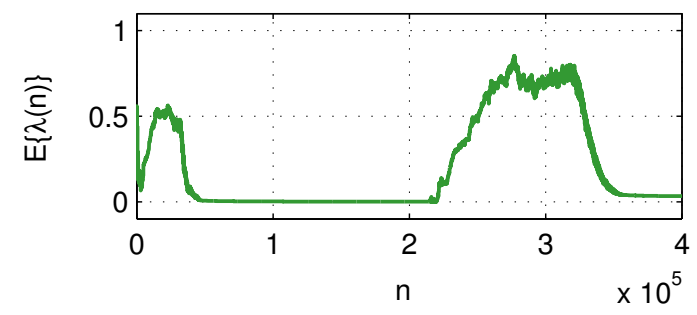

Fig. 8. a) Ensemble-average of the squared absolute value of the decision error for a) MSDD $\left(\mu_{d}=10^{-5}, \rho=0.6\right)$, CMA $\left(\mu_{c}=5 \times 10^{-7}, \mu_{\varphi}=\right.$ $\left.10^{-3}\right)$ and their convex combination C-MSDD-CMA $\left(\mu_{\alpha}=5, \alpha^{+}=4\right)$; $M=23$; 64-QAM, the channel is initially given by the coefficients of Table II and then, it is changed to those of Table I at $n=2 \times 10^{5}$; SNR $=35 \mathrm{~dB}$; mean of 70 independent runs. b) Ensemble-average of $\lambda(n)$.

Communicatioons, vol. 28, pp. 1867-1875, Nov. 1980.

[2] F. C. C. De Castro, M. C. F. De Castro, and D. S. Arantes, "Concurrent blind deconvolution for channel equalization," in Proc. of ICC'2001. IEEE, 2001, vol. 2, pp. 366-371.

[3] S. Chen, "Low complexity concurrent constant modulus algorithm and soft directed scheme for blind equalization," IEE Proceedings - Vision, Image, and Signal Processing, vol. 150, pp. 312-320, Oct. 2003.

[4] S. Chen, T. B. Cook, and L. C. Anderson, "A comparative study of two blind FIR equalizers," Signal Processing, vol. 14, pp. 18-36, Jan. 2004.

[5] J. Karaoguz and S. H. Adarlan, "A soft decision-direct blind equalization algorithm applied to equalization of mobile communication channels," in Proc. of ICC'92. IEEE, 1992, vol. 3, pp. 343.4.1-343.4.5.

[6] S. Chen, S. Mclaughlin, P. M. Grant, and B. Mulgrew, "Multi-stage clustering equaliser," IEEE Transactions on Communications, vol. 43, pp. 701-705, Feb./Mar./Abr. 1995.

[7] J. Arenas-García, M. Martínez-Ramón, A. Navia-Vázquez, and A. R. Figueiras-Vidal, "Plant identification via adaptive combination of transversal filters," Signal Processing, vol. 86, pp. 2430-2438, Sept. 2006.

[8] J. Arenas-García, A. R. Figueiras-Vidal, and A. H. Sayed, "Mean-square performance of a convex combination of two adaptive filters," IEEE Transactions on Signal Processing, vol. 54, pp. 1078-1090, Mar. 2006.

[9] J. Arenas-García and A. R. Figueiras-Vidal, "Improved blind equalization via adaptive combination of constant modulus algorithms," in Proc. of ICASSP'O6. IEEE, 2006, vol. III, pp. 756-759.

[10] M. T. M. Silva and V. H. Nascimento, "Convex combination of blind adaptive equalizers with different tracking capabilities," in Proc. of ICASSP'2007, Apr. 2007.

[11] J. Proakis, Digital Communications, McGraw-Hill, New York, 1995.

[12] A. Heidari and M. Nasiri-Kenari, "A family of simple blind phase recorevy algorithms," in Proc. of ISPACS'2000. IEEE, 2000, vol. 1, pp. 24-28.

[13] C. N. Georghiades, "Blind carrier phase acquisition for QAM constellations," IEEE Transactions on Communicatioons, vol. 45, pp. 1477-1486, Nov. 1997.

[14] L. L. Szczecinski and A. Gei, "Blind decision feedback equalisers, how to avoid degenerated solutions," Signal Processing, vol. 82, pp. 16751693, Nov. 2002. 